\title{
Bewegung ist Leben
}

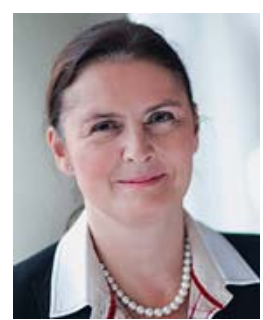

Univ.-Prof. Dr. med. Andrea Meurer

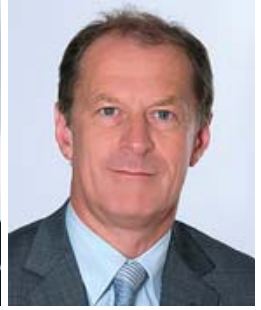

Univ.-Prof. Dr. med. Ingo Marzi

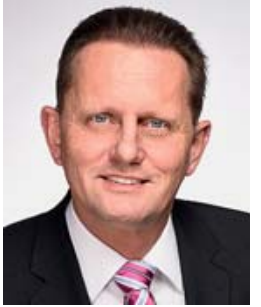

Prof. Dr. med. Alexander Beck
Korrespondenzadresse

Univ.-Prof. Dr. med. Andrea Meurer

Orthopädische Universitätsklinik Friedrichsheim gGmbH

Marienburgstraße 2, 60528 Frankfurt am Main

Andrea.Meurer@friedrichsheim.de

Bibliografie

DOI https://doi.org/10.1055/s-0043-117899

Z Orthop Unfall 2017; 155: 526 @ Georg Thieme Verlag KG

Stuttgart · New York | ISSN 1864-6697

\section{Liebe Leserinnen, liebe Leser!}

Der DKOU ist mit über 12000 Teilnehmern mittlerweile der größte medizinische Fachkongress in Europa und einer der größten Orthopädie- und Traumatologie-Kongresse weltweit. Das diesjährige Motto „Bewegung ist Leben“ unterstreicht die Bedeutung von Bewegung für den menschlichen Organismus. Ohne Bewegung findet Leben nicht statt, regelmäßige Bewegung fördert die Gesundheit nachhaltig und reduziert das Risiko für zahlreiche Erkrankungen. Bewegung erhalten und verlorene Bewegung wieder herstellen ist somit Kernaufgabe des Faches Orthopädie und Unfallchirurgie.

Der DKOU ist für jede Aus- und Weiterbildungsstufe interessant, an diesem seit Jahren bewährten Kongressaufbau wird unverändert festgehalten. Für die jungen Kolleginnen und Kollegen in Weiterbildung wurde ein neues Format „O und U Basics“ eingeführt. Hier wird elementares Grundwissen durch Fachexperten verständlich und ausführlich aufgearbeitet und besprochen. Die Experten finden in den wissenschaftlichen Sitzungen und Expertenrunden zu allen Themenbereichen unseres großen Faches den aktuellen Stand der Wissenschaft. Einen breiten Raum nehmen auch Sitzungen zu Forschungs-, Gesundheits- und Berufspolitik ein. Das diesjährige Gastland, die Vereinigten Staaten von Ame- rika, hat mit zahlreichen Fachgesellschaften zur Gestaltung des Programmes beigetragen. So können wir an jedem Tag 2 parallele Stränge englischsprachiger Sitzungen anbieten. Die zahlreichen Sektionen, Arbeitskreise und Arbeitsgemeinschaften präsentieren sich wie gewohnt am Dienstag, um sich untereinander auszutauschen, aber auch Interessierte für ihre Spezialitäten anzulocken.

Highlights des Kongresses sind unter anderem der Festvortrag im Rahmen der Eröffnungsveranstaltung, dieses Jahr gehalten von dem Dirigenten, Musikproduzenten und Coach Christian Gansch, sowie die Pauwels-Gedächtnis-Vorlesung durch Professor Fritz Hefti.

Ein so großer Kongress wie der DKOU kommt nur durch aktive Mitarbeit zahlreicher beteiligter Menschen und Institutionen zustande. Unser Dank geht an die Fachgesellschaften, an Intercongress und an alle Kolleginnen und Kollegen sowie Mitarbeiterinnen und Mitarbeiter, die dazu beigetragen haben, dass der DKOU 2017 wieder in der gewohnten Qualität stattfinden kann.

Wir freuen uns, Sie in Berlin willkommen zu heißen!

Ihre

Univ.-Prof. Dr. med. Andrea Meurer

Univ.-Prof. Dr. med. Ingo Marzi

Prof. Dr. med. Alexander Beck 